\title{
Microscopic processes during electron cyclotron resonance microwave nitrogen plasma-assisted molecular beam epitaxial growth of GaN/GaAs heterostructures: Experiments and kinetic modeling
}

\author{
Z. Z. Bandića) and T. C. McGill \\ Thomas J. Watson, Sr., Laboratory of Applied Physics, California Institute of Technology, Pasadena, \\ California 91125
}

R. J. Hauenstein ${ }^{\text {b) }}$ and M. L. O'Steen

Department of Physics, Oklahoma State University, Stillwater, Oklahoma 74078

(Received 22 January 1996; accepted 16 April 1996)

\begin{abstract}
A set of $\delta-\mathrm{GaN}_{y} \mathrm{As}_{1-y} / \mathrm{GaAs}$ strained-layer superlattices grown on GaAs (001) substrates by electron cyclotron resonance (ECR) microwave plasma-assisted molecular beam epitaxy (MBE) was characterized by ex situ high resolution X-ray diffraction (HRXRD) to determine nitrogen content $y$ in the nitrided GaAs monolayers as a function of growth temperature $T$. A first order kinetic model is introduced to quantitatively explain this $y(T)$ dependence in terms of an energetically favorable $\mathrm{N}$ for As anion exchange and thermally activated $\mathrm{N}$-surface desorption and surface segregation processes. The nitrogen surface segregation process, with an estimated activation energy $E_{s} \sim 0.9 \mathrm{eV}$ appears to be significant during the GaAs overgrowth of $\mathrm{GaN}_{y} \mathrm{As}_{1-y}$ layers, and is shown to be responsible for strong $y(T)$ dependence. (C) 1996 American Vacuum Society.
\end{abstract}

\section{INTRODUCTION}

In the past few years we have seen a flurry of activity in the development of visible light emitters based on wide band gap refractory semiconductor GaN, related alloys, and heterostructures. ${ }^{1-4}$ Most of the research was concentrated on epitaxial growth techniques of nitrides, ${ }^{5}$ and growth of device structures (LEDs and lasers) on various substrates, ${ }^{6}$ while comparatively little work was done on other materials of potential technological applications, such as mixed anion nitride/arsenide system. ${ }^{7-9}$ Although limited by a small solubility, ${ }^{10}$ even small amounts of $\mathrm{N}$ in GaAs can cause unexpected band gap bowing to infrared, ${ }^{10,11}$ while the small amounts of $\mathrm{As}$ in $\mathrm{GaN}$ can substantially increase valence band spin-orbital splitting which can significantly influence operation of the active layer in the laser structure due to the higher recombination rate. ${ }^{12}$ One of the main difficulties in growing such nitride/arsenide structure, for example, in the electron cyclotron resonance plasma-assisted molecular beam epitaxy (ECR-MBE), is controlling the structural and chemical properties of the interface, which is only possible if important microscopic growth processes are fully understood. We have recently shown ${ }^{7,8}$ that it is possible to produce high quality, fully commensurate $\mathrm{GaN}_{y} \mathrm{As}_{1-y} / \mathrm{GaAs}$ strained-layer superlattices (SLS), grown on the GaAs substrate. Initial high resolution X-ray diffraction (HRXRD) and reflection high energy electron diffraction (RHEED) measurements done on such structures revealed existence of several distinct thermally activated processes, but limited by an unavailability of a suitable quantitative model against which these separate experimental observations could be tested

\footnotetext{
${ }^{\text {a) Electronic mail: zzbssdp.caltech.edu }}$

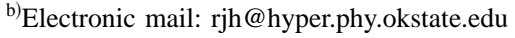

consistently, only semi-quantitative considerations of kinetics and tentative identification of processes have been possible to date. ${ }^{8}$

In this article, we introduce a quantitative model, based on the first order kinetic theory, to examine the initial nitridation (surface $\mathrm{N}$ for As exchange), $\mathrm{N}$ surface desorption, and $\mathrm{N}$ surface segregation processes, observed in ECR-MBE grown $\mathrm{GaN}_{y} \mathrm{As}_{1-y} / \mathrm{GaAs}$ heterostructures, characterized by ex situ HRXRD. Through application of our model to an unusually strong growth temperature dependence of $y$, obtained from X-ray experiments, the existence of a thermally activated $\mathrm{N}$ surface segregation mechanism is conclusively established and quantitatively confirmed. Although previously suggested, ${ }^{8}$ the thermally activated $\mathrm{N}$ surface segregation mechanism could not be conclusively established without adequate analytical model, which is necessary to describe the concurrent, thermally activated $\mathrm{N}$ surface desorption and segregation processes. Activation energies of both surface segregation and desorption processes are numerically estimated, and the impact of the compositional-profile smearing of $\mathrm{N}$, due to the strong $\mathrm{N}$ surface segregation effect, to $\mathrm{GaN} /$ GaAs heteroepitaxy and interface quality is analyzed and discussed.

\section{EXPERIMENTAL DETAILS}

A set of 36-period " $\delta$ - $\mathrm{GaN}_{y} \mathrm{As}_{1-y} / \mathrm{GaAs}$ " superlattices, with a superlattice period consisting of one $\mathrm{GaN}_{y} \mathrm{As}_{1-y}$ monolayer (ML) and 75 GaAs monolayers, was grown on a GaAs (100) substrate as a function of temperature $\left(540-580{ }^{\circ} \mathrm{C}\right)$ in an ECR-MBE system. $\mathrm{GaN}_{y} \mathrm{As}_{1-y}$ monolayers were produced through brief $(4 \mathrm{~s}) \mathrm{N}_{2}$ plasma exposure (nitridation) of an As-stabilized GaAs surface. Nitridation was immediately followed by GaAs overgrowth at a fixed 


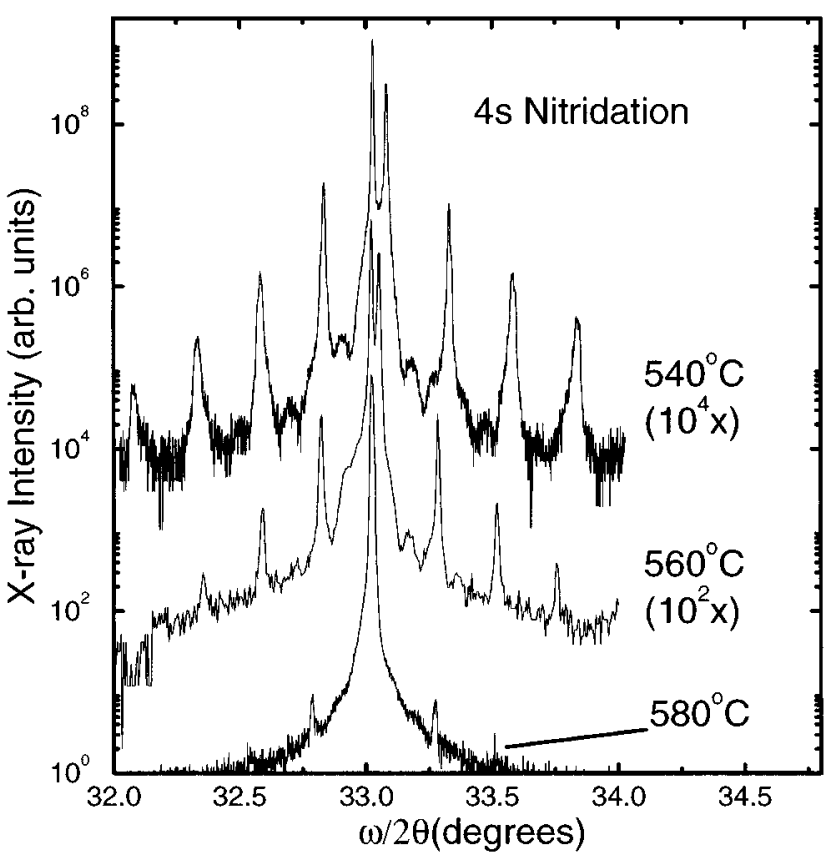

FIG. 1. HRXRD around (004) substrate reflections for $\delta$ - $\mathrm{GaN}_{y} \mathrm{As}_{1-y}$ /GaAs superlattices. Results indicate strong dependence of the rocking curves profiles on growth temperature.

growth rate $(0.75 \mathrm{ML} / \mathrm{s})$ for $100 \mathrm{~s}$. The surface of GaAs is then As-stabilized through exposing to $\mathrm{As}_{2}$ flux for only $30 \mathrm{~s}$ (As-soak). In addition, one superlattice was grown with $6 \mathrm{~s}$ nitridation at $550{ }^{\circ} \mathrm{C}$. Further details about growth system and procedures can be found elsewhere. ${ }^{7,8}$

To determine strain and concentration profile, the entire sample set is then characterized by ex situ HRXRD, with the use of Philips Materials Research Diffractometer (MRD). The standard $\omega / 2 \theta$ scans are performed in the 4-crystal mode using $\mathrm{Ge}(220)$ reflections, around the substrate (004) reflections, while the reciprocal space scans (area scans) around the (115) reflections are done in the same mode, with additional use of a Bonse-Hart collimator in front of the detector. The area scans confirmed that all samples are coherently strained. The resultant $\omega / 2 \theta$ rocking curves for $4 \mathrm{~s}$ nitridations SLS are presented on Fig. $1\left(550^{\circ} \mathrm{C}\right.$ and $570{ }^{\circ} \mathrm{C}$ scans have been omitted for clarity). Results reveal a strong dependence on growth temperature $T$ of the epitaxial structure, indicating a presence of thermally activated microscopic processes. Two-dimensional equivalent alloy compositions $(y)$ are determined from peak positions, and are confirmed through dynamical simulations using Philips High Resolution Simulation (HRS) software package. The agreement typically obtained between experimental rocking curve and simulation is presented on Fig. 2 on the example of the superlattice grown on $550{ }^{\circ} \mathrm{C}$. Figure 3 shows an Arhennius plot of the resultant compositional dependence on growth temperature, $y(T)$, for $4 \mathrm{~s}$ (circles) and $6 \mathrm{~s}$ (diamond) nitridations, including experimental uncertainties in temperature, which represent measured variation over a given wafer (i.e., temperature nonuniformity) due to unintended differences in sample mounting. Although not included in the plot, the absolute errors associated with $\mathrm{GaN}$ composition can be esti-

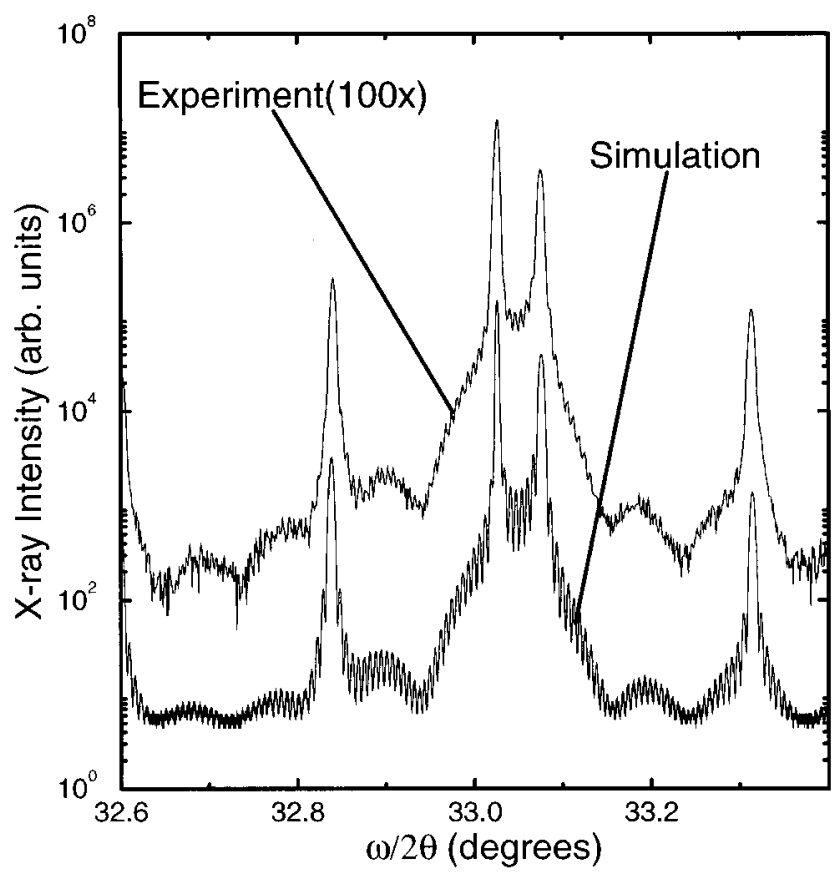

FIG. 2. Typical agreement between experimental rocking curve and HRS siumulated rocking curve.

mated to $\pm 0.5 \%$. These errors are a result of a Vegard's law approximation and uncertainties in numerical values of elastic properties of the $\mathrm{GaN}_{y} \mathrm{As}_{1-y}$ layer.

These results suggest existence of two regimes: $(i)$ doselimited at low temperatures; ( $i i)$ kinetically limited at high

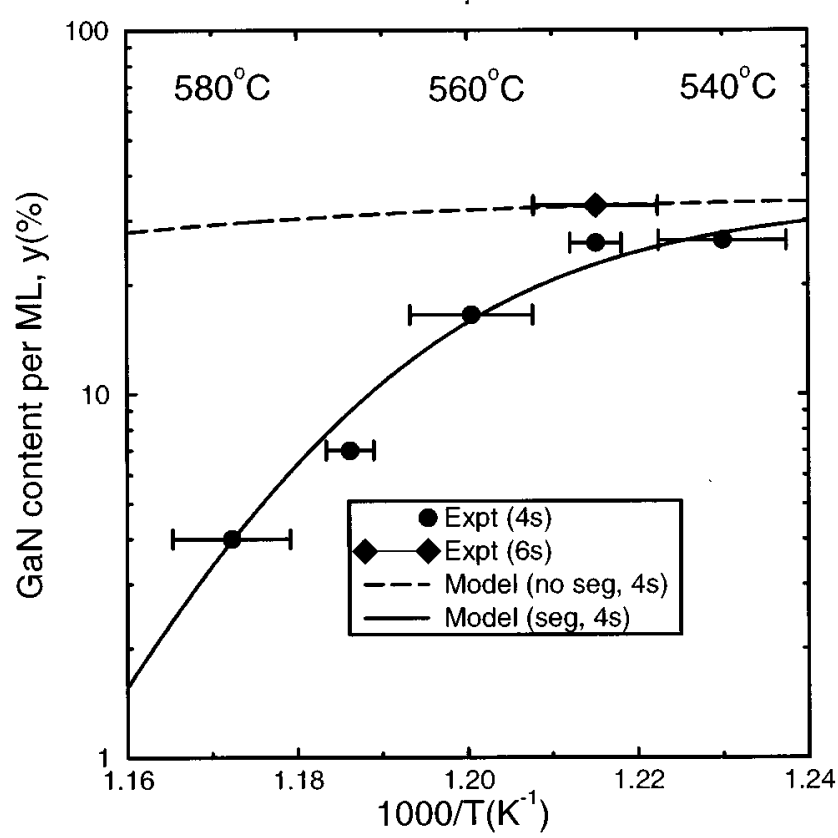

FIG. 3. Growth-kinetics models to explain the strong $y(T)$ dependence observed for ECR-MBE grown $\delta$ - $\mathrm{GaN}_{y} \mathrm{As}_{1-y} / \mathrm{GaAs}$ strained-layer superlattices. Experimental data points are obtained through HRXRD measurements for $4 \mathrm{~s}$ (circles) and $6 \mathrm{~s}$ (diamond) nitridations. The dashed curve represents the model neglecting $\mathrm{N}$ surface segregation [Eq. (2)], while the solid curve represents the model including segregation effects [Eq. (8)]. 
temperatures, with unexpectedly strong fall-off in $y$ with increasing growth temperature.

\section{KINETICAL MODELING}

For the purpose of understanding the physical origin of this $y(T)$ dependence, we have developed a first order kinetic model which explicitly incorporates three phases of our particular SLS growth sequence: (i) nitridation; (ii) overgrowth, and (iii) As-soak, as described in Sec. II.

\section{A. Nitridation}

The net rate of surface nitridation is determined by two physical processes which have been previously identified: (i) $\mathrm{N}$-for-As surface anion exchange ( $\mathrm{N}$ gain), and (ii) surface $\mathrm{N}$ desorption ( $\mathrm{N}$ loss). These processes can be analyticaly modeled, in the first order in $\mathrm{N}$ surface compostion $y$ as

$$
\frac{d y}{d t}=s \Phi(1-y)-\frac{y}{\tau_{d}} .
$$

The first term on the right hand side of Eq. (1) corresponds to the $\mathrm{N}$ gain on the As-stabilized GaAs surface due to the energetically favorable anion exchange. This process is modeled as being proportional, in the first order, to the flux $\Phi$ of incident physically activated $\mathrm{N}$; to the fraction of available As surface sites to be exchanged with $\mathrm{N}$, and to an overall efficiency factor $s$ (units of area), which in general might depend on surface chemistry, strain, and temperature, but is approximated as constant over the temperature and composition range of interest, for the purpose of our simplified model. The second term in this equation, $-y / \tau_{d}$, represents the $\mathrm{N}$ loss due to surface desorption. It can be simply modeled in terms of thermally activated rate constant $\tau_{d}^{-1}=\tau_{0 d}^{-1} \exp \left(-E_{d} / k T\right)$, with activation energy $E_{d}$. Such a process has been directly observed in our RHEED measurements of nitrided GaAs surfaces, and estimates for $E_{d}(\sim 2.1$ $\mathrm{eV}$ ) have been previously reported. ${ }^{7,8}$

If we define an "effective dosing rate" $r \equiv s \Phi$, the solution to Eq. (1) for an initially N-free (As-stabilized) surface can be written as

$$
y\left(t_{\text {exp }}\right)=y_{\mathrm{ss}}\left[1-\exp \left(-t_{\exp } / \tau\right)\right],
$$

where $y_{\mathrm{ss}} \equiv r \tau, \tau \equiv\left(\tau_{d}^{-1}+r\right)^{-1}$, and $t_{\exp }$ is the nitrogen plasma exposure time. If Eq. (2) is considered in the limit of low temperatures so that desorption rate $\tau_{d}^{-1}$ is negligible, then $y \rightarrow 1$ would be obtained. This reflects the assumption that As atoms from the As-enriched (100) GaAs surface, when exposed to $\mathrm{N}$ plasma, will be completely exchanged for $\mathrm{N}$ atoms in the steady state, since $\mathrm{N}$ terminated surface has lower surface free energy, due to a much stronger $\mathrm{Ga}-\mathrm{N}$ than $\mathrm{Ga}-\mathrm{As}$ bond (heats of formation $6.81 \mathrm{eV}$ and $5.55 \mathrm{eV}$, respectively $\left.{ }^{13}\right)$. Although the essential nitridation kinetics is well described by Eq. (2) without unnecessary mathematical complexity, it ignores the subsequent stages of growth of our superlattices, and therefore cannot be used to model directly our experimental results. This can be seen in Fig. 3, where the dashed curve represents the plot of Eq. (2) taking $t_{\exp }=4 \mathrm{~s}$ and using our RHEED based experimental desorp- tion parameters, $E_{d}=2.1 \mathrm{eV}$ and $\tau_{d}=7 \mathrm{~s}$ at $592^{\circ} \mathrm{C} .{ }^{7,8} \mathrm{In}$ addition, the dosing rate $r$ is estimated to be approximately $0.1 \mathrm{~Hz}$ from experimental values of $y$ at $550{ }^{\circ} \mathrm{C}$ for $4 \mathrm{~s}$ and 6 $\mathrm{s}$ nitridations, such that Eq. (2) describes properly the doselimited (low temperature) regime of growth.

\section{B. GaAs overgrowth-N segregation}

Inspection of Fig. 3 clearly reveals that the processes of anion exchange and $\mathrm{N}$ surface desorption alone result in the weak fall-off of the model (dashed) curve, and thus cannot account for the strong $y(T)$ dependence in the kinetically limited growth regime. Also, even $\mathrm{N}$ surface desorption during the short monolayer deposition time $\left(t_{\mathrm{ML}}\right)$ required to bury (and freeze-in) the nitrided $\mathrm{GaN}_{y} \mathrm{As}_{1-y}$ layer cannot result in significant $\mathrm{N}$ loss. To overcome this, we hypothesize that thermally activated $\mathrm{N}$ surface segregation process, described with the rate constant $\tau_{s}^{-1}=\tau_{0 s}^{-1} \exp \left(-E_{s} / k T\right)$, is occurring concurrently with $\mathrm{N}$ desorption, during GaAs overgrowth. ${ }^{8}$ This assumption is supported qualitatively by our experimental observation that $\mathrm{GaN}$-related $(3 \times 3)$ surface reconstruction proceeds faster to GaAs $(2 \times 4)$ reconstruction at a lower substrate temperature during GaAs overgrowth, which is consistent with freeze-out of a thermally activated $\mathrm{N}$ segregation process.

Let us assume that (1) segregation occurs from the first subsurface layer upward to the current surface layer; (2) desorption occurs from all currently exposed surfaces, i.e., the current growth surface and the current partially exposed first sub-surface layers; and (3) the segregation and desorption processes are statistically independent, and are occurring concurrently. Also, let $S_{n}$ represent a relative GaN mole fraction in monolayer $n$, where by definition the content of the nitrided layer at the end of nitridation process is normalized to unity, $S_{0}(t=0) \equiv 1$. Under these assumptions, we can relate the $\mathrm{N}$ content of monolayers $n$ and $n+1$ through

$$
S_{n+1}(0)=\left[S_{n}(0)-S_{n}\left(t_{\mathrm{ML}}\right)\right] \frac{\tau_{s}^{-1}}{\tau_{s}^{-1}+\tau_{d}^{-1}} .
$$

Equation (3) describes the fraction of lost $\mathrm{N}$ $\left[S_{n}(0)-S_{n}\left(t_{\mathrm{ML}}\right)\right]$, which segregates rather then desorbs, and becomes the nitrogen content of the next surface layer. Since layer $n$ loses its $\mathrm{N}$ content due to both $\mathrm{N}$ desorption and segregation, during the time interval $0 \leqslant t \leqslant t_{\mathrm{ML}}, S_{n}(t)$ decays exponentially as $\exp \left(-t / \tau_{\|}\right)$, where $\tau_{\|}^{-1}$ $\equiv\left(\tau_{s}^{-1}+\tau_{d}^{-1}\right)$. Using this expression in Eq. (3) leads us to

$$
S_{n+1}(0)=S_{n}(0)\left[1-\exp \left(-t_{\mathrm{ML}} / \tau_{\|}\right)\right] \frac{\tau_{d}}{\tau_{\mathrm{tot}}},
$$

where $\tau_{\text {tot }} \equiv \tau_{s}+\tau_{d}$. It is obvious from Eq. (4) that $S_{n}(0)$ represents geometrical progression. Now, similarly to Eq. (3) we can define $\delta_{n}$ as the amount of initial material lost through desorption from layer $n$ :

$$
\delta_{n} \equiv\left[S_{n}(0)-S_{n}\left(t_{\mathrm{ML}}\right)\right] \frac{\tau_{s}}{\tau_{\mathrm{tot}}}
$$


Therefore, the total loss of $\mathrm{N}$ from one superlattice period during overgrowth would be

$$
L=\sum_{n=0} \delta_{n},
$$

which is simply a sum of a geometric progression. Finally, the total amount of the original GaN content retained within one period of a superlattice is $R=1-L$, or straightforwardly

$$
R=\frac{\tau_{\text {tot }}}{\tau_{d}+\tau_{s} e^{t} \mathrm{ML}^{/} \tau_{\|}} .
$$

It is important to note that the expression for $R$ given in Eq. (7) gives the correct limiting behaviour in describing the loss of $\mathrm{N}$ during the GaAs overgrowth, in the limits of no segregation, or no desorption. If there is desorption, but no segregation, then the only opportunity for $\mathrm{N}$ loss would be before nitrided layer is completely buried in GaAs; i.e., $R=\exp \left(-t_{\mathrm{ML}} / \tau_{d}\right)$, which is obtained directly from Eq. (7) in the limit $\tau_{s} \rightarrow \infty$. Similarly, if there is segregation, but no desorption, then $R$ must be unity, since the only possible mechanism for $\mathrm{N}$ loss is desorption process. In that case, where $\tau_{d} \rightarrow \infty$, one would obtain $R \rightarrow 1$, as it should be.

\section{RESULTS AND DISCUSSION}

Using the "segregation correction factor" from Eq. (7), it is clear that in order to consistently incorporate $\mathrm{N}$ surface segregation into our model, Eq. (2) must be replaced by

$$
y\left(t_{\mathrm{exp}}\right)=y_{\mathrm{ss}}\left[1-\exp \left(-t_{\exp } / \tau\right)\right] \times\left[\frac{\tau_{\text {tot }}}{\tau_{d}+\tau_{s} e^{t_{\mathrm{ML}} / \tau_{\|}}}\right] .
$$

Thus, Eq. (8) represents the final result of our model and quantitatively describes the growth sequence (nitridation/ overgrowth/soak) of our $\delta$-GaN $\mathrm{As}_{1-y} / \mathrm{GaAs}$ superlattices, taking into account microscopic processes of anion exchange, desorption, and segregation, and is plotted as the solid curve in Fig. 3. This curve is calculated using identical dosing $(r)$ and desorption $\left(E_{d}\right.$ and $\left.\tau_{0 d}\right)$ parameter values which were used in Eq. (2) to obtain the dashed curve, while adjusting at the same time two segregation parameters, $E_{s}$ and $\tau_{0 s}$. Nonlinear least-squares fit to the experimental data points for $4 \mathrm{~s}$ nitridations finally results in the estimate for $\mathrm{N}$ surface segregation energy equal to $0.9 \mathrm{eV} \pm 30 \%$. Obviously, from Fig. 3, it is seen that with the corrected Eq. (8) very good agreement with experimental data is obtained, which confirms our $\mathrm{N}$ surface segregation hypothesis.

Additionally, the compositional-profile smearing of $\mathrm{N}$ along the growth direction would be expected as a natural consequence of the segregation process, which is confirmed in preliminary cross sectional scanning tunneling microscopy (STM) images of one of our superlattices. ${ }^{14}$ Unfortunately, such profile smearing effects would tend to limit the abruptness of the GaN/GaAs interface. However, it is possible that smearing effects might be controlled through some of the
MBE techniques which have been succesfully used in the problem of dopant-profile smearing in MBE-grown $\mathrm{Si}^{15}$ Also, the cross-sectional STM as a direct imaging technique might provide another way to determine $E_{s}$, since our model predicts distribution of nitrogen in the superlattice period $\left(S_{n}(0)\right)$, as a function of $\mathrm{N}$ surface activation energy $E_{s}$ and growth temperature $T$.

\section{SUMMARY}

In conclusion, a simple, but plausible kinetic model have been developed to conclusively establish and quantitatively explain some of the microscopic processes observed in ECRMBE grown $\delta$ - $\mathrm{GaN}_{y} \mathrm{As}_{1-y} / \mathrm{GaAs}$ strained layer superlattices. Particularly, the strong $y(T)$ dependence, obtained through ex situ HRXRD of our superlattices, is fully understood in terms of energetically favorable surface anion exchange and combined, thermally activated $\mathrm{N}$ surfacesegregation/surface-desorption. Finally, our model predicts for the first time numerical estimates of the kinetic parameters associated with $\mathrm{N}$ surface-segregation process, which appears to be significant under typical GaN/GaAs ECR-MBE growth conditions.

\section{ACKNOWLEDGMENTS}

This work was supported by Advanced Research Project Agency, and monitored by Office of Naval Research under Grant No. N00014-92-J-1845. Additionally, two of us (R.J.H. and M.L.O.) wish to acknowledge the support of Air Force Office of Scientific Research under Contract Nos. F49620-93-1-0211 and F49620-93-1-0389, and the support of the Advanced Research Projects Agency, monitored through the Army Research Office under Contract No. DAAH04-94-G-0393.

${ }^{1}$ S. Nakamura, M. Senoh, S. Nagahama, N. Iwasa, T. Yamada, T. Matsushita, H. Kiyoku and Y. Sugimoto, Jpn. J. Appl. Phys. 35, 74 (1996).

${ }^{2}$ S. Nakamura, M. Senoh, N. Iwasa, and S. Nagahama, Appl. Phys. Lett. 67, 1868 (1995).

${ }^{3}$ I. Akasaki, H. Amano, S. Sota, H. Sakai, T. Tanaka, and M. Koike, Jpn. J. Appl. Phys. 34, 1517 (1995).

${ }^{4}$ H. Sakai, T. Koide, H. Suzuki, M. Yamaguchi, S. Yamasaki, M. Koike, H. Amano, and I. Akasaki, Jpn. J. Appl. Phys. 34, L1429 (1995).

${ }^{5}$ W. Gotz, N. M. Johnson, J. Walker, D. P. Bour, H. Amano, and I. Akasaki, Appl. Phys. Lett. 67, 2666 (1995).

${ }^{6}$ S. N. Mohammad, A. A. Salvador, and H. Morkoç, Proc. IEEE 83, 1306 (1995).

${ }^{7}$ R. J. Hauenstein, D. A. Collins, X. P. Cai, M. L. O'Steen, and T. C. McGill, Appl. Phys. Lett. 66, 2861 (1995).

${ }^{8}$ R. J. Hauenstein, D. A. Collins, M. L. O'Steen, Z. Z. Bandić, and T. C. McGill, Mater. Res. Soc. Symp. Proc. 388, 259 (1995).

${ }^{9}$ M. Sato, Jpn. J. Appl. Phys. 34, 1080 (1994).

${ }^{10}$ J. Neugebauer and C. G. Van de Walle, Phys. Rev. B 51, 10568 (1995).

${ }^{11}$ S. Sakai, Y. Ueta, and Y. Terauchi, Jpn. J. Appl. Phys. 32, 4413 (1993).

${ }^{12}$ Z. Z. Bandić and T. C. McGill (unpublished).

${ }^{13}$ CRC Handbook of Chemistry and Physics, edited by R. C. West (Chemical Rubber, Boca Raton, FL, 1987), p. E102.

${ }^{14}$ R. M. Feenstra (unpublished).

${ }^{15}$ H. Jorke, Surf. Sci. 193, 569 (1988). 